\title{
Optical calibration of the ELT adaptive mirror M4: design, alignment and verification of the interferometric test tower
}

\author{
Giorgio Pariani ${ }^{1}$, Matteo Tintori ${ }^{2}$, Runa Briguglio ${ }^{3}$, Marco Xompero ${ }^{3}$, Armando Riccardi ${ }^{3}$, Daniele \\ Gallieni $^{2}$, Roberto Biasi ${ }^{4}$, Elise Vernet ${ }^{5}$, Marc Cayrel $^{5}$ \\ ${ }^{1}$ INAF - Osservatorio Astronomico di Brera, via E. Bianchi 46, 23807 Merate, Italy \\ ${ }^{2}$ A.D.S International, via Roma 87, 23868 Valmadrera, Italy \\ ${ }^{3}$ INAF - Osservatorio Astrofisico di Arcetri, largo E. Fermi 5, 50125 Firenze Italy \\ ${ }^{4}$ Microgate, via Stradivari 4, 39100 Bolzano, Italy \\ ${ }^{5}$ ESO Headquarter, Karl Schwarzschild strasse 2, D-85748 Garching, Germany
}

\begin{abstract}
The Optical Test Tower (OTT) is the facility for the interferometric calibration of the M4 Unit (M4U) -the ELT sixsegment, $2.4 \mathrm{~m}$ flat deformable mirror- now in the final design phase by AdOptica. It is composed by two main subsystems: a large aperture setup, which accomplishes a macro-stitching approach where each mirror segment is flattened and co-phased to the other petals, and a sub-aperture setup to certify the mirror quality at the high spatial frequency scales. The accurate calibration of the interferometric cavity is of capital importance to meet the tight coflattening accuracy of the segmented adaptive mirror. We will analyze the critical aspects affecting the optical performances of the OTT, in particular: the absolute measurement of the optical alignment, the calibration strategy we foresee for the main optical components, the nulling of $1.5 \mathrm{~m}$ interferometric cavity, and the management of the drift of the optical surfaces. The M4U project is led by the Italian consortium AdOptica under an ESO contract.
\end{abstract}

Keywords: ELT, M4, adaptive mirror, optical calibration, test setup

\section{INTRODUCTION}

The M4 adaptive Unit (M4U) of the ELT provides the telescope the ground layer wavefront correction and fast steering capabilities for the real-time atmospheric turbulence and wind shaking suppression, in addition to static aberrations compensation ${ }^{1}$. The mirror is $2.4 \mathrm{~m}$ in diameter, segmented into six petals, and shaped by approximately 6000 voice-coil actuators. The M4U is now under the Final Design Phase by the consortium AdOptica (Microgate, A.D.S. International, and INAF as sub-contractor) under the ESO contract. INAF, for the expertise acquired in past activities with the LBT $^{2}$ and $\mathrm{VLT}^{3}$ deformable mirrors, is in charge for the design of the optical test setup and for the support to the M4U optical tests. With the LBT and VLT the optical calibration procedure for single shell mirrors was defined (a comprehensive description may be found in the literature ${ }^{4}$ ). In the M4U case, the complexity of the optical test design is particularly high for the tight specifications imposed by the contractor along with the segmented nature of the mirror. We started to address the calibration of a segmented system with the M4 Demonstration Prototype, a two segments, 222 actuators, with particular attention to segments management, correction of differential alignment, and co-phasing ${ }^{5}$.

To accomplish the M4U calibration, we designed a dedicated facility, called Optical Test Tower (OTT), composed by two main subsystems: a Large Aperture Interferometric setup (LAI), able to image the whole mirror segment, to perform the flattening of the single shells and the co-phasing between the petals, and a Sub-Aperture Interferometric setup (SAI), used to certify the mirror quality at the high spatial frequency scales. In order to meet the tight co-flattening accuracy of the M4 mirror, both systems require a very accurate calibration of the interferometric cavity in terms of alignment errors, residual high order errors, thermal drifts and turbulence behavior. After the description of the OTT systems, we will analyze the critical aspects affecting the optical performances of the OTT, in particular: the absolute measurement of the optical alignment, the calibration strategy we foresee for the main optical components, and the nulling of the interferometric cavity.

*giorgio.pariani@brera.inaf.it 


\section{OTT TEST SYSTEMS}

Two optical configurations are implemented on the OTT. In the Large Aperture interferometric mode (LAI) the entire M4U segment is imaged with a $1440 \mathrm{~mm}$ beam; the Sub-Aperture interferometric mode (SAI) is instead based on a 100 $\mathrm{mm}$ test beam to provide the high spatial frequency verification of the M4U. Such separated test solutions are requested to improve the measurement resolution and accuracy. A further test device will be employed in the LAI mode, called Sensor of Phase Lag (SPL), to remove the lambda ambiguity of the interferometric measurements and to allow the cophasing of adjacent shells. A rotation of the test beam on the M4 axis and its translation in a plane normal to M4 are required in both measurement modes to test the whole mirror surface. Figure 1 shows the test beam in the LAI and SAI measurement modes and Table 1 the M4U optical calibration procedure with the associated OTT system used. A detailed description of each test can be found in the literature ${ }^{4}$

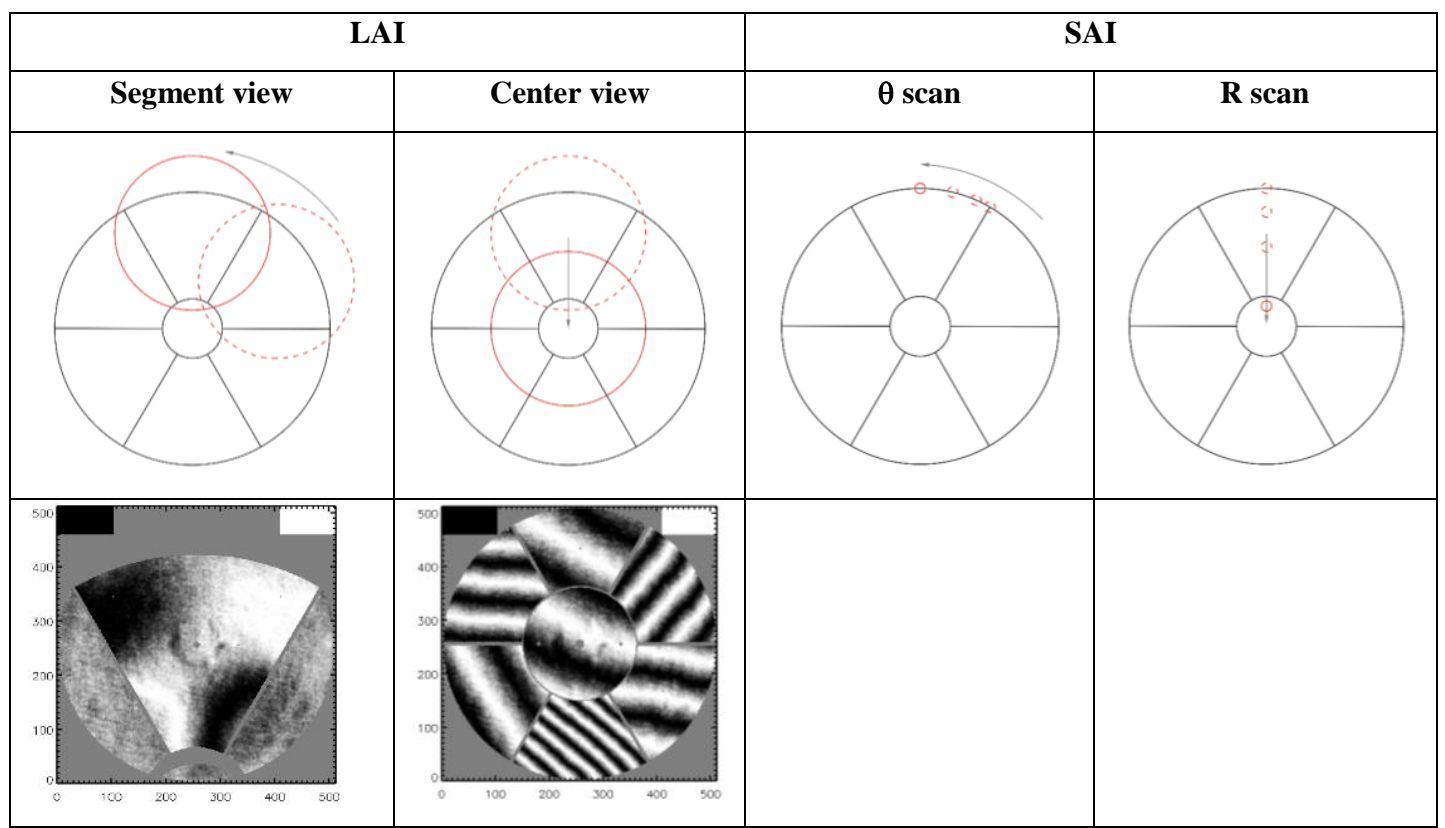

Figure 1: test beam (red) and M4 in the LAI and SAI measurement modes. The simulated interferograms in the LAI mode are obtained with the simulation tool $8 \mathrm{~s}^{6}$.

Table 1: M4U test procedure with the corresponding OTT measurement modes.

\begin{tabular}{|l|l|l|}
\hline Action & Comment & Mode \\
\hline preliminary flattening & $\begin{array}{l}\text { identification of a set of actuator commands to have the whole } \\
\text { mirror surface within the interferometer capture range }\end{array}$ & LAI, segment view \\
\hline mirror modes collection & measurement of modal and zonal influence functions & LAI, segment view \\
\hline flattening procedure & $\begin{array}{l}\text { correction of the high order modes; low order modes, like } \\
\text { piston and tip-tilt, are not corrected at this point }\end{array}$ & LAI, segment view \\
\hline capacitive sensors calibration & internal metrology (capacitive sensors) are optically calibrated & LAI, segment view \\
\hline differential tip/tilt removal & $\begin{array}{l}\text { local tip/tilt between segments is measured and removed before } \\
\text { proceeding to co-phasing }\end{array}$ & LAI, center wiew \\
\hline lambda ambiguity removal & $\begin{array}{l}\text { differential piston between segments is reduced to a fraction of } \\
\text { fringe }\end{array}$ & SPL \\
\hline mirror phasing & performed with the interferometer & LAI, center view \\
\hline requirements verification & performed on the flattened and co-phased M4 mirror & LAI, SAI \\
\hline
\end{tabular}




\section{LARGE-APERTURE INTERFEROMETRIC MODE (LAI)}

\subsection{Test Setup}

The LAI mode is designed to cover the single shell with a beam size of $1440 \mathrm{~mm}$. This limits the beam diameter while avoiding sub-aperture stitching. The optical scheme is based on a beam expander setup, realized with a large collimator, to test M4 at normal incidence. The Parabolic Mirror (PAR) is placed with the optical axis vertical, facing up. Actually, the main constraint for the optical design is to avoid beam vignetting from the interferometer head. Given the geometry of the interferometer beam, a Relay System (RS) is necessary to move the interferometer, placed horizontally, outwards the main beam. The test scheme is reported in Figure 2.

The clear aperture of the PAR is $1440 \mathrm{~mm}$, as the dimension of the testing beam, and its radius of curvature is $7 \mathrm{~m}$ (f/2.43). The RS is composed by three lenses and a folding mirror (a modified $4 \mathrm{f}$ design), mounted along with the interferometer on the same support. Its main purpose it to generate a beam with the correct $\mathrm{f} / \#$ to feed the parabolic mirror, and relay the interferometer pupil so that the M4 mirror is focused on the interferometer detector. In this way, the vignetting of the RS is limited to a small circle of $30 \mathrm{~mm}$ in diameter in the center of the collimated beam, which is the area of interest of one actuator only. The test setup include also a Reference Mirror (RM), a 600 mm perfectly flat mirror, that has to be inserted in the interferometric cavity upon request, to verify the alignment of the optical system. In order to freeze both mechanical vibrations and air turbulence, we selected a vibration insensitive Twyman-Green interferometer (4D Technology), able to collect frames with an exposure time down to $30 \mathrm{us}$ at a rate faster than $25 \mathrm{~Hz}$. This is of particular importance for the test schedule, especially in the differential measurements regime, when hundreds of frames has to be collected in the shortest time possible to freeze the slow cavity drifts ${ }^{3}$.
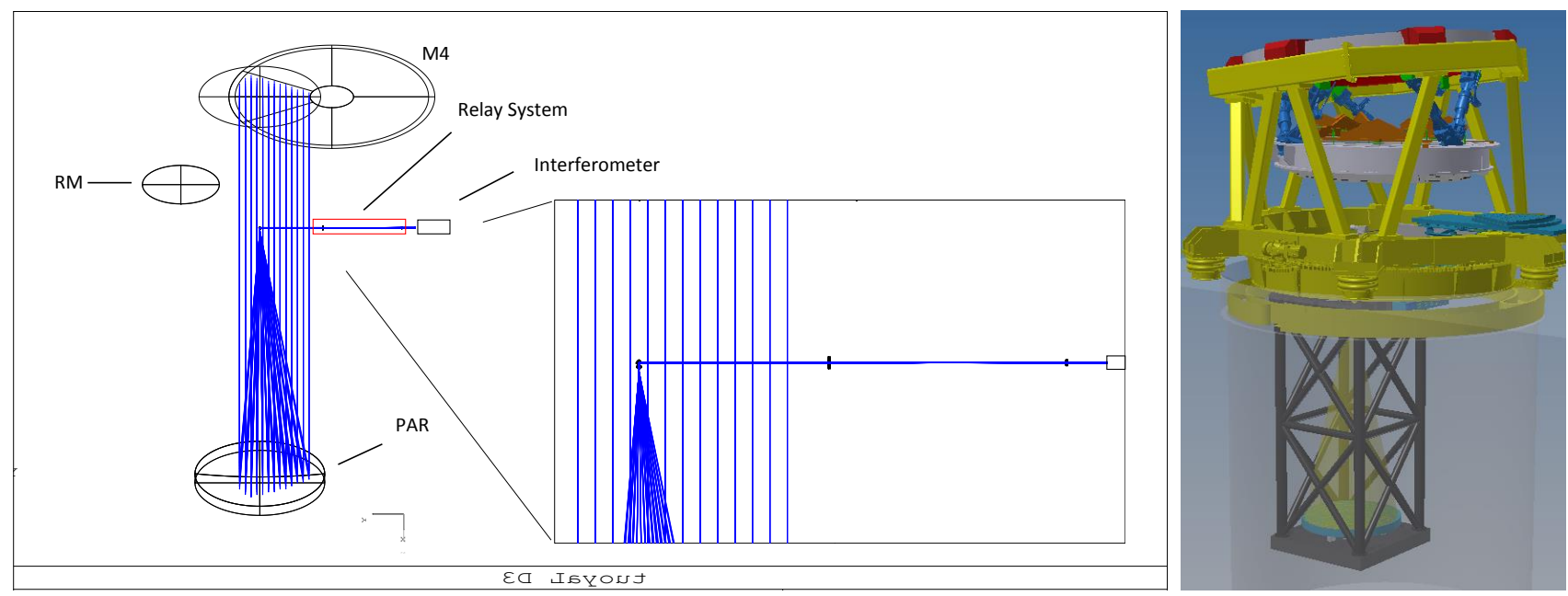

Figure 2: LAI optical test setup. Optical design (left) and mechanical implementation (right).

\subsection{Movements and Degrees of Freedom (DoF) distribution}

In order to implement the different measurement modes the parts are moved as follows:

- M4, mounted on its structure and hexapod, is free to position in the six DoFs

- the structure with the RS and PAR rotates and translates below M4 (on the M4 optical axis), moving the OTT cavity below the single shells (segment view) or in the central region of M4 (center view)

- the reference mirror slides inside the OTT cavity, and a balancing mass is moved outside, accordingly.

The DoFs were distributed among the different optical elements according to their position and accessibility to allow their preliminary manual alignment and the active alignment of the cavity to compensate for thermal and mechanical drifts during normal operation (Table 2). The decentering of the PAR is not actively controlled, since can be 
compensated by a rotation of the PAR around its focal point followed by a rotation of the M4 of the same amount. The pupil shift induced by this procedure is easily tolerated.

Table 2: alignment DoF of the OTT ( $\mathrm{z}$ is aligned as the local optical axis).

\begin{tabular}{|l|l|l|l|l|}
\hline & RS & PAR & RM & M4 \\
\hline Preliminary alignment & $\mathrm{dx}, \mathrm{dy}, \mathrm{dz}, \mathrm{rx}, \mathrm{ry}$ & $\mathrm{dx}, \mathrm{dy}, \mathrm{dz}, \mathrm{rx}, \mathrm{ry}$ & $\mathrm{dx}, \mathrm{dy}$ & $\mathrm{dx}, \mathrm{dy}, \mathrm{rx}, \mathrm{ry}$ \\
\hline Active DoF & - & $\mathrm{dz}, \mathrm{rx}, \mathrm{ry}$ & $\mathrm{rx}, \mathrm{ry}$ & $\mathrm{dx}, \mathrm{dy}, \mathrm{rx}, \mathrm{ry}$ \\
\hline
\end{tabular}

\subsection{Imaging performances}

Imaging performances (image quality and registration) are of great importance both during the calibration phase and the verification phase of the mirror. Apart common elements, the testing of deformable mirror has some peculiarities that are considered in the following sections.

\section{Image resolution}

In general, the image resolution shall be sufficient to describe features at the maximum spatial frequency of interest. In our case, the 1200x1200 pixel camera of the selected interferometer gives an effective spatial resolution on the M4 mirror of $1.8 \mathrm{~mm} / \mathrm{pixel}$, corresponding to a sampling of 18 pixel/actuator, which is large enough to sample the actuator influence functions.

\section{Geometrical distortions}

The test setup is designed to provide a flat wavefront at the interferometer pupil. The mapping from test mirror to the interferometer has been verified to be linear, hence no remapping is necessary to obtain the undistorted image of the M4 mirror. Fiducials placed on the PAR will be used to properly align the PAR map for subtraction. Field curvature and distortion of the imaging beam are reported in Figure 3. The maximum distortion is $0.02 \%$, corresponding to $0.3 \mathrm{~mm}$ on M4 (to be compared with the pixel scale of $1.8 \mathrm{~mm} / \mathrm{pixel}$ ).

\section{Image shift for the out of null condition}

When collecting the mirror modal basis or the Zernike shapes, the OTT cavity is out of the null condition, and map misplacements as well as retrace errors must be avoided. These issues are usually not present in standard interferometric measurements, where the measurement are performed in the null condition. To consider this point, we started from the fact that the interferometer is a coherent imaging system, where the light propagation can be described using only one ray per point of the object ${ }^{7}$. For design purposes, the imaging system can be treated using an infinitesimal bundle of rays, originating from each point on the surface under test (M4), and propagating to the interferometer exit pupil. The width of the ray bundle has been defined using the stop at the focal point of the PAR, which size has been determined as the maximum position of the focal point for all the admissible slopes of the deformable mirror during, for example, influence function measurements or the Zernike test.
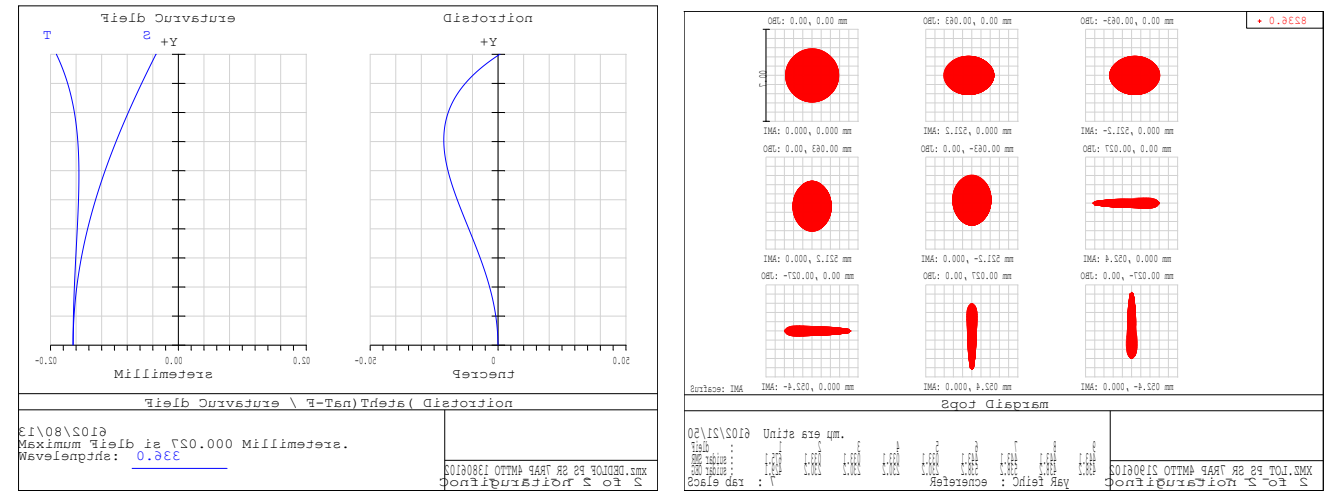

Figure 3: field curvature and distortion of the imaging beam (left) and spot diagrams of the imaging beam for different pupil positions for 20 arcsec M4 slope (right). 
We verified that the RMS spot size is always below half pixel, meaning that one point on M4 will strike the same pixel on the detector for any possible slope of the M4U, ensuring good image registration of the test mirror (Figure 3). A slight degradation to the pixel size was noted only for the Nyquist condition at the edges of the pupil.

\section{Phase distortions}

Phase smoothing is caused by diffraction, producing a change in the high spatial frequency components of the wavefront, i.e., high frequency phase data may be filtered out. According to the literature ${ }^{8,7}$, phase smoothing can be treated with the Talbot imaging equations in the small-phase approximation. In practice, the propagation of a small wavefront aberration at a given frequency is propagated from the ideal image plane to the real image plane with the Talbot propagation, to define the transfer function of the optical system. The difference between the ideal and real image planes for any field point is obtained from the field curves, that do not use information about the size and position of the stop, but are calculated using the Coddington relations. With the proposed model we calculated the optical Transfer Function (TF) of the LAI setup considering a maximum defocus of $0.2 \mathrm{~mm}$ as function of the wavefront aberration frequency over the interferometer aperture (Figure 4 left). The instrument TF depends on the interferometer and on the reconstruction method, and has been derived from the literature ${ }^{9}$ with a linear extrapolation to a $1200 \times 1200$ pixel sensor from the 1000x1000 pixel case with the ' $2 \times 2$ ' reconstruction method (Figure 4 center). The system transfer function is then calculated by multiplying the optical TF and the instrument TF and is reported in Figure 4 (right). At the $80 \mathrm{~mm}$ spatial scale (18 cycles/aperture) and at the interactuator scale (50 cycles/aperture) the transfer function is almost unitary. This means that the system gain is unitary for all the spatial scales that can be controlled by the mirror actuators. A good information transfer ( $\mathrm{TF}>0.5$ ) can be observed up to about 360 periods/aperture, corresponding to a spatial resolution of $4 \mathrm{~mm}$ period.
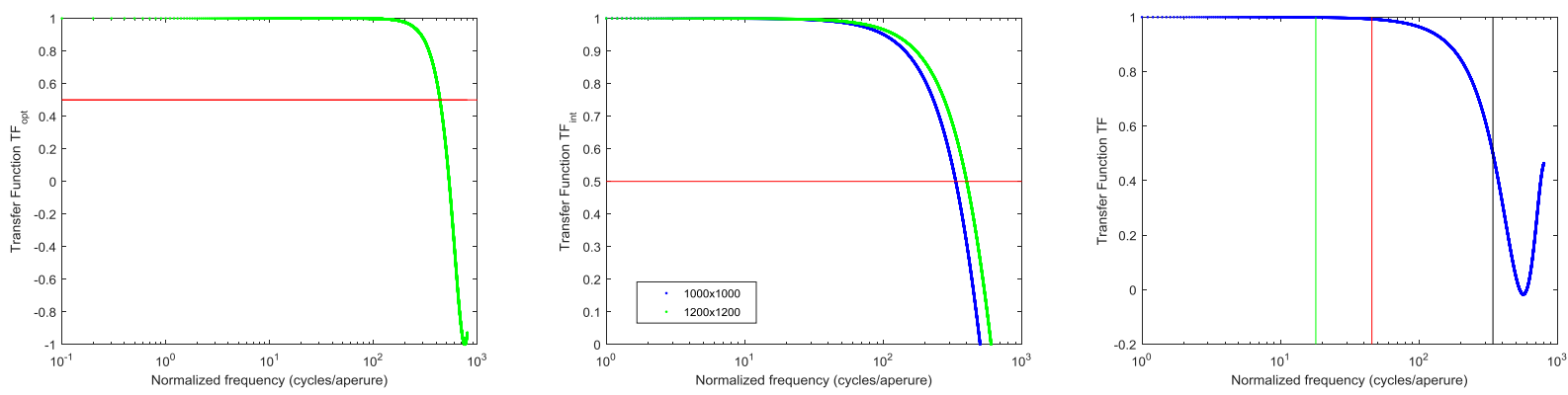

Figure 4: optical TF due to phase smoothing (left); interferometer TF for the $2 \mathrm{x} 2$ reconstruction method as function of the sensor pixel size, in the case of 1000x1000 and 1200x1200 pixel detector (center); system TF, the vertical lines correspond to $80 \mathrm{~mm}$ scale (green), actuator scale (red) and $4 \mathrm{~mm}$ scale (black).

\subsection{Sensor of Phase Lag (SPL)}

The SPL will be used to locally resolve the phase ambiguity in the differential piston between adjacent shells, before the co-phasing with the OTT in the LAI mode. A similar device was developed for the Phase-B of the M4 Demonstration Prototype $^{10}$, showing uncertainties in the differential piston better than $30 \mathrm{~nm} \mathrm{PV}{ }^{11}$. Six SPLs will be mounted onto the M4U and slide under the mirror to measure each shell gap. The basic principle of the SPL consists into the illumination with a collimated beam of the shell gap. The reflected light is then re-focalized onto a detector, and the diffraction pattern recorded. The analysis of the diffraction spot allows the determination of the local piston between the two shells (Figure 5 left). Among the different techniques to retrieve the piston signal, we will consider the multi-lambda approach obtained with a liquid crystal tunable filter with selectable bandwidth. It provides a continuously tunable center wavelength between 420 and $730 \mathrm{~nm}$ with a user-selectable bandwidth setting of few tens of nanometers FWHM. The tunable filter approach was already used in the past along with a pyramid wavefront sensor for the phasing of a segmented mirror ${ }^{12,13}$. The differential piston can be retrieved by scanning the shell gap with the different wavelengths, without any mechanical movement of the shells, by fitting the PSF barycenter as function of the illumination wavelength. In particular, the piston signal $\mathrm{S}$ has a sinusoidal dependence on the inverse of the wavelength, in the form of ${ }^{12}$ 


$$
S=A+B \sin \left(2 \pi \frac{2 \delta}{\lambda}\right)
$$

where $\delta$ is the physical differential piston between the shells (see Figure 5 right). The working limits of this technique are the minimum and the maximum detectable steps. The minimum physical step that can be measured is defined by the extreme values of the wavelength applicable in the sweep, because a minimum phase variation is required to fit the signal function. Supposing that a quarter of period can be fitted, we obtain:

$$
\Delta \varphi=2 \pi 2 \delta_{\min } \frac{\lambda_{2}-\lambda_{1}}{\lambda_{1} \lambda_{2}}=\frac{\pi}{4}
$$

and a minimum detectable piston difference

$$
\delta_{\text {min }}=\frac{1}{16} \frac{\lambda_{1} \lambda_{2}}{\lambda_{2}-\lambda_{1}}=60 \mathrm{~nm}
$$

On the contrary, the capture range, which is the maximum detectable step, can be calculated from the finest wavelength variation ${ }^{12}$. When the piston is large, the sine modulation is very dense, and to avoid aliasing in the detection we have to suppose a maximum phase variation of a quarter of period. Accordingly, considering a minimum step of $10 \mathrm{~nm}$ and a wavelength of $420 \mathrm{~nm}$, we obtain

$$
\delta_{\text {max }}=\frac{1}{8} \frac{\lambda_{1} \lambda_{2}}{\lambda_{2}-\lambda_{1}} \cong 20 u m
$$

which is sufficient to correct the differential piston calibrated by the electromechanical tests performed on the M4 before the optical calibrations.

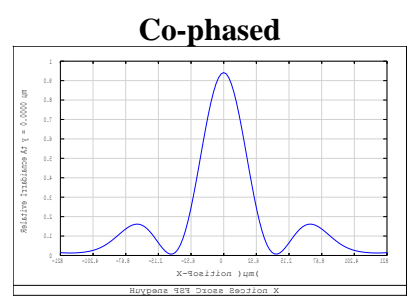

$150 \mathrm{~nm}$ piston

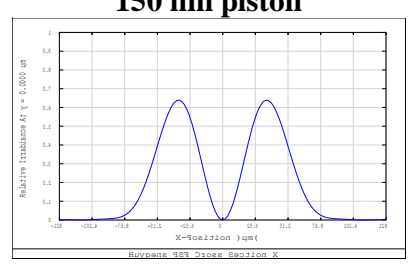

$100 \mathrm{~nm}$ piston

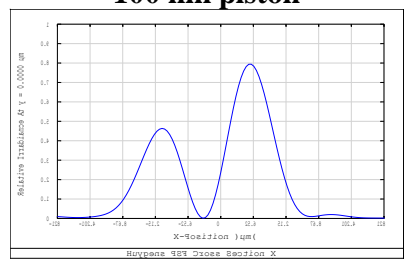

$250 \mathrm{~nm}$ piston

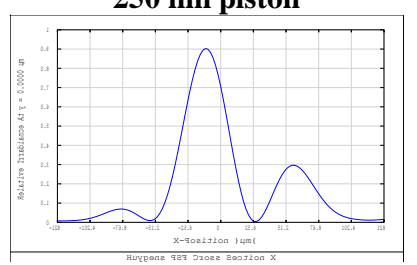

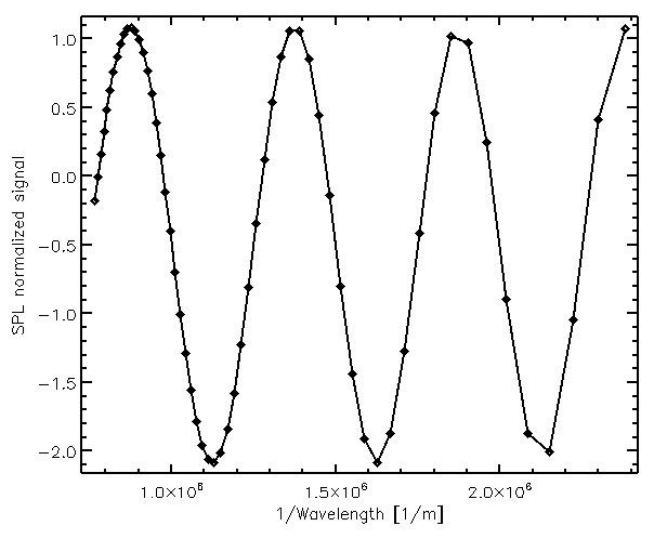

Figure 5: diffraction PSFs cross sections at $600 \mathrm{~nm}$ as function of the differential piston between the shells (left) and detected signal on the SPL as function of the light wavelength.

The optical layout of the SPL (Figure 6) consists into a point-like source (a mono mode optical fiber) collimated to a size of 10-12 mm by an achromatic doublet. After reflection on the shell gap, the focal extraction is increased by means of an achromatic negative doublet and focalized on the CCD camera. The system f/\# is 70, diffraction limited between 400 to $700 \mathrm{~nm}$. The main driver of the optical design is a good sampling of the diffraction pattern. In this direction, a large system f/\# produces a larger spot size, but also a bigger setup. On the other hand, the beam size on the shell plane must be large enough compared to the shell gap in order to limit the number of the side peaks in the spot size. Accordingly, the optical design has been optimized for a $2 \mathrm{~mm}$ gap to contain the system width within $500 \mathrm{~mm}$. A camera with 3.75 
micron pixel pitch will provide a sampling of 20 pixel/peak in the direction perpendicular to the slit, sufficient to correctly identify the peak position and intensity. The mechanical design, composed by off the shelf component, is also shown in Figure 6.

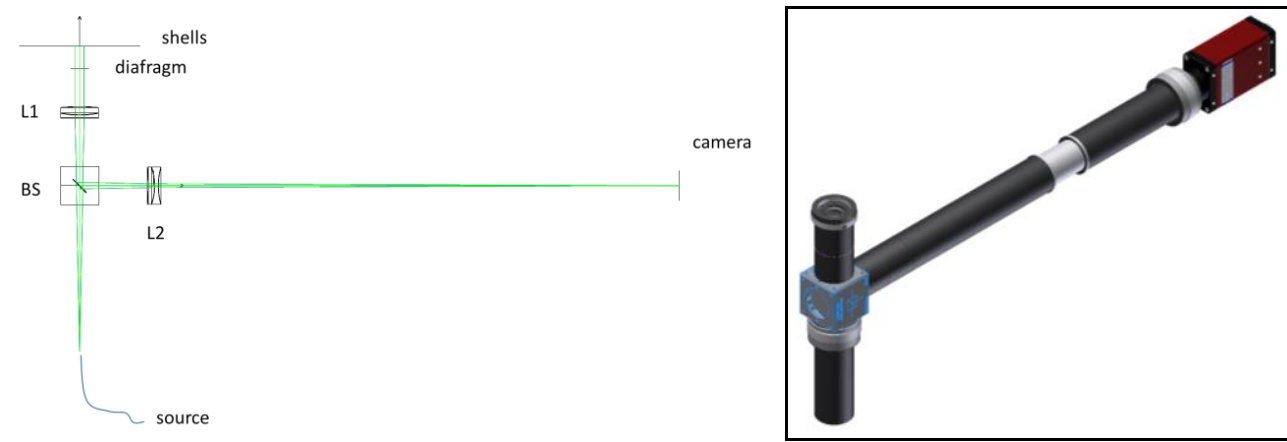

Figure 6: SPL optical and mechanical designs.

\subsection{Sub-Aperture Interferometric mode (SAI)}

The quality of the M4U at the small spatial scales of the inter-actuator distance will be certified with a second measurement setup, consisting of an interferometer with a collimated beam of $100 \mathrm{~mm}$ in diameter. After the flattening and co-phasing of the M4U with the LAI setup, the secondary interferometer setup SAI is moved across the optical area to measure the $100 \mathrm{~mm}$ patches.

The interferometric test setup is composed by a refractive beam expander, as shown in Figure 7. We considered two positive lens doublets, working at f/7.5. The beam is optimized to produce a pupil at the interferometer exit pupil position of $6.5 \mathrm{~mm}$. Accordingly, the resolution on the M4U is $0.150 \mathrm{~mm} /$ pixel. Producing such a large beam with very high WF quality is very tough, especially for the optical manufacturing of the elements, therefore we decided to design a simpler system, to be calibrated with a test flat before its use to guarantee the nanometer level accuracy. Calibration will be performed by sliding the RM on top of the SAI setup before the measurement on the M4U. If no absolute techniques are used, its ultimate precision is limited by the quality of the RM over the $100 \mathrm{~mm}$ patches. Since the RM is much larger than the SAI beam, a quasi-absolute calibration procedure can be implemented, by averaging multiple phasemaps taken at different positions of the RM. It is important to underline that no high frequency errors (pixel-to-pixel) are introduced during calibration, since no rescaling or repositioning between the measurement and calibration maps is necessary. Moreover, misalignments of the optical setup will introduce only low order modes and, according to the measurement procedure, the local tip-tilt is subtracted from the whole map before estimation of the requirement on the interactuator patches. The estimated error budget after calibration, calculated on the inter-actuator distance diameter, is below $5 \mathrm{~nm}$ RMS.

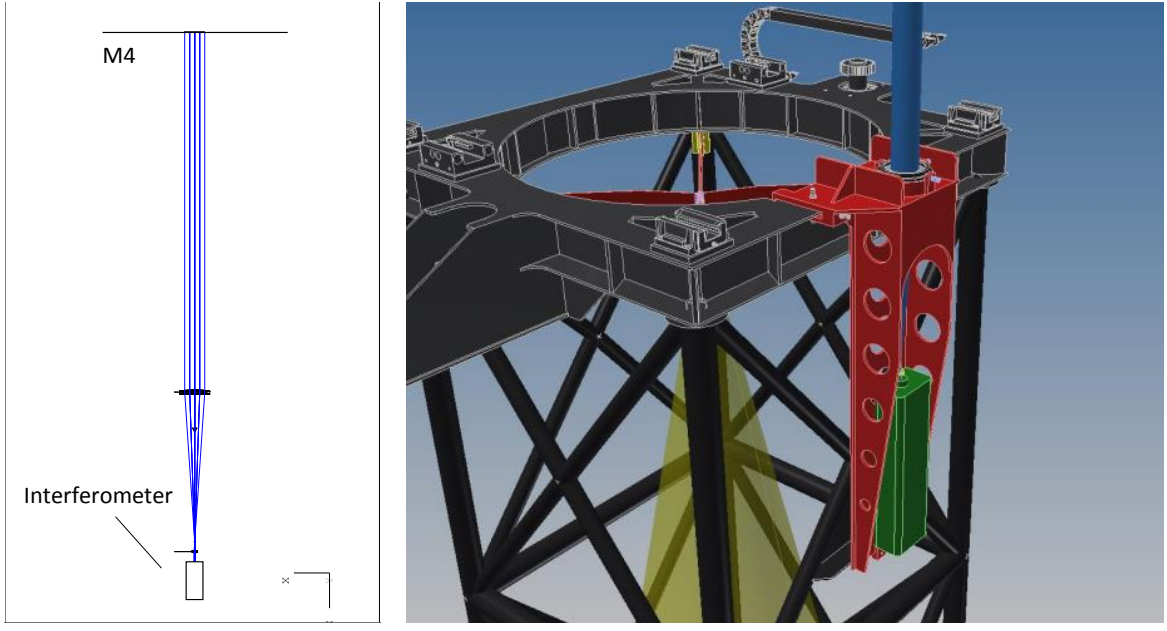

Figure 7: SAI test setup optical and mechanical designs. 


\section{LAI CAVITY CALIBRATION}

In order to reach the required level of accuracy in the M4U test and to calibrate the absolute flat command of the mirror, the LAI cavity shall be characterized before or during operation, to subtract the errors due to optics manufacturing (in double pass), alignment and drifts. The procedure to calibrate the OTT cavity will be as follows:

- calibration of the main optical components: optical elements (PAR, RS, RM) are measured before the installation in the OTT, to give reference phasemaps to be subtracted from the OTT cavity. Methods and errors connected to the RS and PAR calibration will be described.

- absolute measurement of the optical alignment: by inserting the RM in the OTT cavity, alignment aberrations are measured on the RM and optical element position adjusted accordingly to null the misalignment; if residual errors are still present, the corresponding wavefront will be stored for the analytical subtraction.

- nulling of the OTT interferometric cavity: the reference phasemaps (containing optics errors and misalignment errors) are registered on the OTT phasemaps thanks to markers on the optics, and their subtraction from the OTT wavefront will give the absolute cavity wavefront. Errors connected to this process (e.g., misregistrations) will be presented.

Other critical aspects that may affect the optical performances of the OTT are the management of the slow drift of the optical surfaces and the cavity retrace error when the measurement is performed out of the null condition, i.e., when the mirror is not flat.

\subsection{Calibration of the main optical components}

The M4U commands are calibrated with the LAI in different OTT configurations and the results are verified both with the LAI and SAI. It follows therefore that the optics manufacturing affects the requirement verification twice.

- $\quad$ as flattening offset: the flattening command minimizes the current WFE within the actuator space and includes therefore the optics print-through associated with the current OTT configuration. Such actuator command offset is preserved when changing the OTT configuration or switching to SAI. This offset is due to the manufacturing precision of the optical elements, which can be minimized by the subtraction of the offset WFE from the OTT cavity WFE.

- $\quad$ as measurement error, i.e., the manufacturing accuracy of the optical elements is an unseen and affects directly the OTT wavefront.

In LAI mode, the error is due to the accuracy of the RS, of the RM and of the PAR.

\section{Relay system calibration}

The Random Ball Test (RBT) for calibrating interferometer transmission spheres has been recently reported in the literature ${ }^{14}$. It is a clever idea to calibrate interferometer aberrations using a statistical average of measurements of a sphere placed at the interferometer focus. If the sphere is sampled in non-overlapping regions, the topography of these regions is uncorrelated and independent. Accordingly, the average of these measurements cancels out the surface figure of the sphere and returns the interferometer aberrations, with an accuracy depending on the maps averaged, but ultimately better than the interferometer repeatability ${ }^{15}$. The RS calibration will be performed both in laboratory after the RS alignment and in the OTT after the RS/PAR installation. A three degrees of freedom mounting (dx, dy, dz) has been designed to properly align the reference sphere/reference ball versus the RS, and an automatic system to rotate the sphere is under evaluation. The image degradation due to edge diffraction on the margin of the pupil and phase smoothing due to the out of focus condition of the ball was limited using the central part of the clear aperture of the interferometer (about $90 \%$ of the CA) and a large reference ball. The calibration image can be as accurate as $1 \mathrm{~nm}$ RMS, with no image registration error since no remapping is required.

\section{Parabolic mirror calibration}

The calibration of the PAR is more challenging, since there are no easy methods to characterize its shape with a quasiabsolute method in the OTT and the mirror is used in double pass. For these reasons, the PAR optical quality is the worst offender in the OTT error budget, even considering the best polishing capabilities of nowadays. The idea is hence to limit as much as possible any error introduced by mechanical and thermal stresses. A ceramic glass with low CTE is considered, to limit thermal deformations. Interfaces mounted on the mirror (e.g., three pads at $120^{\circ}$ ) and kinematics supports are used to hold the mirror in the polishing process and in the OTT as well, to avoid mechanical stressed due to 
the mounting. The mirror will also be polished and tested in the same gravity orientation as in the OTT. A further test of the mirror is foreseen before its installation in the OTT, to get the actual mirror shape at the operating temperature, to be subtracted from the OTT cavity. In this operation, even if markers are present on the mirror for the PAR map registration as respect to the OTT cavity, errors may be introduced by small misalignments. On top of these, we need a so tight testing accuracy of the M4U such that the testing accuracy of the mirror has to be taken into consideration. Accordingly, the PAR affects the OTT cavity with the following contributions:

- manufacturing precision: it is responsible for offset of the OTT cavity, which are aberrations and features given by the polishing process. These errors are measured and stored on the PAR calibration phase-map, and are effectively subtracted unless errors in the map registration is present. The spatial frequencies of this error depends on the manufacturing tool, and are peculiar of the used production method.

- manufacturing accuracy: the PAR is polished against a null optics, either a null lens or CGH, until the desired surface is obtained. This means that the accuracy of the test setup is polished into the PAR, producing a static surface offset. The PAR offset is propagated in the OTT cavity, according to the number of reflections of the PAR, and is not seen by calibration phasemap of the PAR.

The contributions to the residual RMS at the different spatial frequencies are crucial to evaluate the error propagation. Actually, only the residual errors within the actuator space are dangerous for the mirror calibration, since are corrected by mirror commands. Error at smaller spatial frequencies are not an issue for the mirror calibration, but may be a problem if high frequency requirement verifications are requested.

To generate the PAR surface map we considered a Montecarlo approach for the low spatial frequencies errors (LO) -up to the Zernike standard term 91- added to template maps of high frequency errors (HO) -up to the resolution of the PAR map measurement. The contributions of the two errors have been varied as well as their overall impact to analyze their influence on the OTT wavefront; a shift of two pixels has been considered as misregistration error in the PAR map subtraction for the calculation of the precision term. Table 3 reports the results of the propagation of the PAR polishing precision and accuracy on the OTT cavity. This analysis is useful to have the feeling of the contributions off each term, but it not directly the PAR error budget. In fact, the generation of the PAR manufacturing requirements has to pass through the projection of these errors into the actuator space.

Table 3: propagation of the PAR errors to the OTT and M4

\begin{tabular}{|c|c|c|c|c|c|c|}
\hline \multicolumn{7}{|c|}{ PAR manufacturing precision } \\
\hline \multicolumn{3}{|c|}{ PAR surface error RMS (nm) } & \multirow{2}{*}{$\begin{array}{l}\text { WFE RMS on } \\
\text { RM (nm) }\end{array}$} & \multirow{2}{*}{$\begin{array}{l}\text { M4 surface } \\
\text { slope } \mathrm{RMS} \\
\text { (asec) }\end{array}$} & \multirow{2}{*}{$\begin{array}{c}\text { M4 surface } \\
\text { curvature on } 80 \\
\text { mm scale }(1 / \mathbf{k m})\end{array}$} & \multirow{2}{*}{$\begin{array}{l}\text { OTT wavefront } \\
\text { (tilt removed) on } \\
31 \mathrm{~mm} \text { scale } \\
\text { (STD of } 95 \% \text { of } \\
\text { the sample) }\end{array}$} \\
\hline TOTAL & LO & HO & & & & \\
\hline 30 & 29.8 & 3.3 & 15 & 0.27 & $1 / 230$ & 9.3 \\
\hline 30 & 28.3 & 10 & 27 & 0.78 & $1 / 105$ & 28 \\
\hline 60 & 59.9 & 3.3 & 30 & 0.29 & $1 / 125$ & 10 \\
\hline 60 & 59.2 & 10 & 33 & 0.79 & $1 / 91$ & 28 \\
\hline \multicolumn{7}{|c|}{ PAR manufacturing accuracy } \\
\hline & & 2.7 & 13 & 0.19 & $1 / 150$ & 8.6 \\
\hline & 5.3 & & 21 & 0.07 & $1 / 204$ & 1.8 \\
\hline
\end{tabular}

In general, HOs are the main contributors to the error propagation, both for the precision and accuracy terms, in all the evaluated parameters. LOs are actually effectively rejected by the calibration process (in this case, the larger the misregistration error the worst the error subtraction). The slope term and the wavefront at the actuator scale depends only on the HO term, while the wavefront at the RM scale and the curvature has an intermediate behavior. Considering the PAR polishing precision, the LOs has practically no influence even if in this case no map shift and subtraction is performed, since their global value is very low, while HOs have a comparable influence as in the polishing precision case. In fact, HOs are not rejected with the calibration process. These table was very useful for the determination of the PAR manufacturing requirements and forced us to reject the LAI mode for the verification of the mirror at the highest spatial scales, that will be performed with the SAI setup. 


\subsection{Measurement of the optical alignment}

The optical alignment of the OTT cavity gives low order wavefront errors that, if not measured, can be corrected by the M4 mirror itself giving a wrong flat command. Therefore, we need an absolute reference for the cavity alignment to measure the alignment aberrations with sufficient accuracy to feedback the OTT components position. The flat mirror RM will act as reference. PAR and RM are moved in three and two DoFs, respectively, to compensate for defocus, tip/tilt and coma as measured on the RM, which may be inserted and extracted to perform the alignment or to view the entire M4U segment. The M4U is aligned by its hexapod to null the tilt on the segment mask.

We simulated the alignment procedure of the cavity with the simulation tool $8 \mathrm{~s}^{6}$ in the presence and absence of vibration noise in the OTT. In the absence of vibration noise, we measured a fit accuracy better than $3 \sigma$, with no correlation with the static offset added. This observation may be explained considering that the aperture of the RM $(600 \mathrm{~mm})$ is large enough to correctly sample the alignment modes (coma and power) of the whole beam $(1440 \mathrm{~mm})$. The results in the case of vibration noise are shown in Figure 8. The simulated OTT configuration with the RF at the alignment position $0.3 \mathrm{~m}$ from the M4U center is presented. The simulated interferometer fringes and the associated phase map show that focus and coma were added (displacing the parabola from the nominal position). Also in this case, we verified a good fitting of the alignment terms (focus and coma) on the RM, for two different positions of the RM into the OTT cavity and different mirror sizes. We observe a better fit for the larger RM, but in any case the maximum fit accuracy error is below $0.2 \%$.
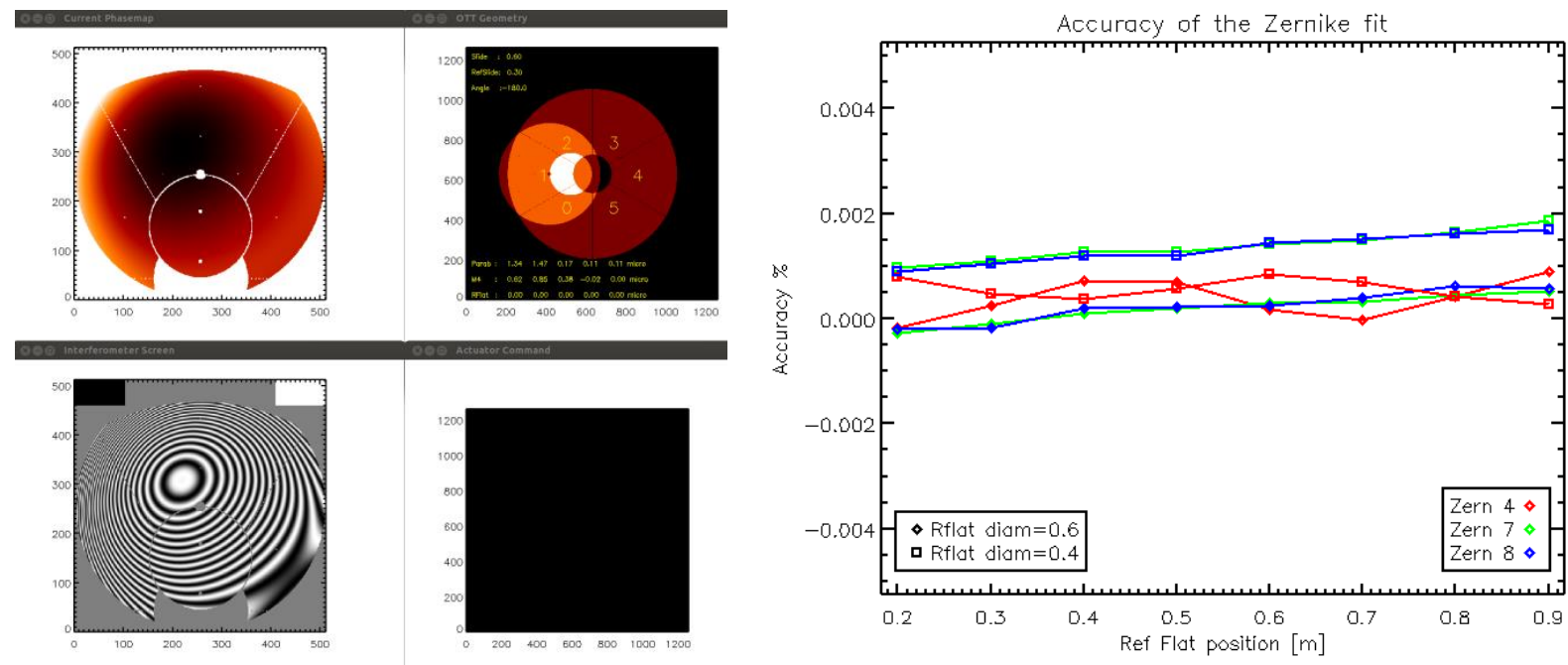

Figure 8: Simulated OTT configuration for a PAR misalignment (left) and the error on estimation of Z4, Z7, Z8 evaluated on RM of different sizes and positions into the cavity (right).

\subsection{Management of the slow drift of the optical surfaces}

Drifts in the OTT alignment, obtained with the help of the RM, are not a problem for differential measurements, which are performed at $25 \mathrm{~Hz}$ and are intrinsically insensitive to low alignment variations. In order to reduce the error in absolute measurements, a time average will be performed during several minutes. In this time frame, the perfect OTT alignment shall be maintained to avoid alignment errors, which is the case of a perfectly stable room and structure temperature. If the temperature is drifting (the order of magnitude is $1 \mathrm{~K}$ over the time span between two calibrations with the RM) a complementary approach to reduce the thermal sensitivity of the alignment errors of about ten times is needed in order to meet the alignment requirements.

Tilt and focus are the main alignment errors that are sensitive to temperature variations, which are at the first approximation, linearly dependent on the temperature. Of course, the available DoFs at the PAR and M4 level are enough to correct for these aberrations. A metrology system is therefore foreseen to retrieve the optical components position as function of the temperature, and synthetically calculate the alignment errors at any time to be subtracted individually by each OTT wavemap. Such metrology shall have an incremental accuracy of better than 1/10th of a 
degree. This seems achievable with the use of PT1000 sensors conveniently placed all along the structure. It must be remembered that the performance of such a metrology system shall last over the time span between two calibrations. On the other side, a thermal model able to predict the optical displacements based on FEA and on the metrology has to be implemented and verified in the OTT. At the first glance, we verified on a large steel structure in a normal working environment that the temperature variation gradients over the structure, during the time span estimated between two calibrations, is negligible. This is due to the large thermal capacity of the structure and the good thermal conductivity, meaning that a large heat is required to change the structure temperature, but the heat is suddenly stabilized along the structure. Figure 9 (left) shows the temperature behavior of the air and the steel structure in the workshop environment over a period of more than 7 days. The temperature variation has a standard deviation of about $0.177 \mathrm{~K} / 90$ min while the steel structure has a standard deviation of about $0.067 \mathrm{~K} / 90 \mathrm{~min}$, which are enough to guarantee the stability of the OTT during the time span between two calibrations.

\subsection{Re-trace error}

The retrace error is produced when the interferometer measures the M4U with large aberrations (tilt, power or higher orders), which is the case of the Zernike test, when a large stroke Zernike shape (up to 20 um PV wavefront) is applied to the mirror and the optical verification is requested. Before analyzing the retrace error, we verified that the number of interferometer fringes when a Zernike term is applied is much lower than the Nyquist frequency, to guarantee a good fringe sampling and no aliasing. Then, once demonstrated the possibility to record the different interferograms, we verified the accuracy of the calibration to the system retrace errors. The OTT WFE ( $\left.W F E_{\text {OTT }}\right)$ was simulated and compared to the theoretical WFE ( $W F E_{c o m}$, twice the applied command for the double reflection on M4, and sampled on the single shell), and the retrace error calculated according to

$$
E r r=\frac{R M S\left(W F E_{\text {OTT }}-W F E_{\text {com }}\right)}{R M S\left(W F E_{\text {com }}\right)} \cdot 100
$$

Results are reported in Figure 9 (right) for the first 11 Zernike Fringe modes of the M4 (20 um PtV WF and 6 um PtV WF for Z9) showing in all cases values below $0.3 \%$ of the applied command.
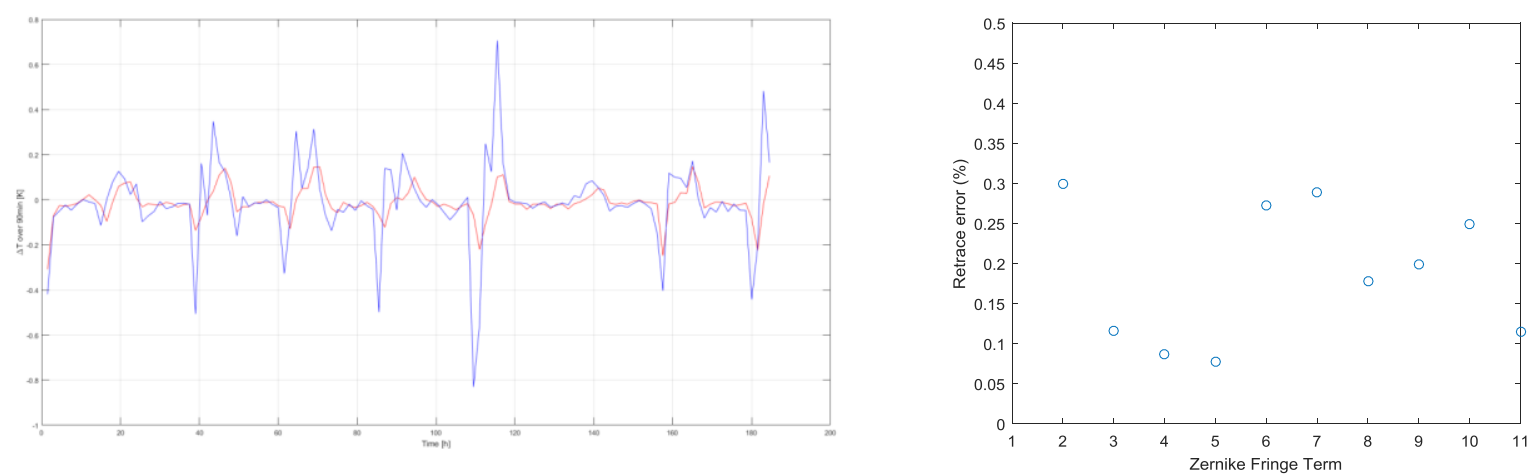

Figure 9: temperature gradient in 90 min for (blue) the air and (red) the steel structure (left); retrace error computed on a single shell as a fraction of the WF command applied to the segment surface (right).

\section{CONCLUSIONS AND PERSPECTIVES}

The OTT is a fundamental piece in the path between the construction and the commissioning of the M4U. This design shows the effective solutions that can be used to characterize large flat and segmented deformable mirrors with voicecoil technology. The presented mechanical solutions, alignment procedures and error mitigation strategies should allow the calibration of the M4U within the tight requirements given by the contractor and hopefully will be used as template for other large deformable mirror test towers. 


\section{REFERENCES}

[1] Vernet, E., Cayrel, M., Hubin, N., Mueller, M., Biasi, R., Gallieni, D.., Tintori, M., "Specifications and design of the E-ELT M4 adaptive unit,” B. L. Ellerbroek, E. Marchetti, and J.-P. Véran, Eds., 844761-844761-844768 (2012).

[2] Riccardi, A., Xompero, M., Briguglio, R., Quirós-Pacheco, F., Busoni, L., Fini, L., Puglisi, A., Esposito, S., Arcidiacono, C., et al., "The adaptive secondary mirror for the Large Binocular Telescope: optical acceptance test and preliminary on-sky commissioning results," Proc. SPIE 7736, 77362C-77362C-12 (2010).

[3] Briguglio, R., Xompero, M., Riccardi, A., Andrighettoni, M., Pescoller, D., Biasi, R., Gallieni, D., Vernet, E., Kolb, J., et al., "Optical calibration and test of the VLT deformable secondary mirror," 3rd AO4ELT Conf. Adapt. Opt. Extrem. Large Telesc.(May), 0-7 (2013).

[4] Briguglio, R., Xompero, M.., Riccardi, A., Optical calibration of adaptive mirrors, O. A. di A. INAF, Ed. (2016).

[5] Briguglio, R., Pariani, G., Marco Xompero., Riccardi, A., Andrighettoni, M., Biasi, R., Tintori, M.., Gallieni, D., "Optical calibration of the M4 prototype toward the final unit," Adapt. Opt. Extrem. Large Telesc. 4 - Conf. Proc. (2015).

[6] Briguglio, R., Pariani, G., Xompero, M., Riccardi, A., Tintori, M., Lazzarini, P.., Spanò, P., "8s, a numerical simulator of the challenging optical calibration of the E-ELT adaptive mirror M4,” Proc. SPIE - Int. Soc. Opt. Eng. 9909, 1-11 (2016).

[7] Zhao, C.., Burge, J. H., “Imaging aberrations from null correctors,” Proc. SPIE 6723, 67230L-67230L-12 (2007).

[8] Zhou, P., Shu, Y., Zhao, C.., Burge, J. H., "Diffraction effects for interferometric measurements due to imaging aberrations," Opt. Express 20(4), 4403-4418 (2012).

[9] Kimbrough, B. T.., Millerd, J., "The spatial frequency response and resolution limitations of pixelated mask spatial carrier based phase shifting interferometry,” Proc. SPIE 85706(1), 77900K (2010).

[10] Molinari, E., Tresoldi, D., Toso, G., Spano, P., Mazzoleni, R., Riva, M., Riccardi, A., Biasi, R., Andrighettoni, M., et al., "The optical tests for the E-ELT adaptive mirror demonstration prototype," 773632-773632-773638 (2010).

[11] Noethe, L.., Adorf, H. M., "Optical measurements of phase steps in segmented mirrors - fundamental precision limits," Optics, 23 (2006).

[12] Pinna, E., Esposito, S., Puglisi, A., Pieralli, F., Myers, R. M., Busoni, L., Tozzi, A.., Stefanini, P., "Phase ambiguity solution with the Pyramid Phasing Sensor," Proc. SPIE 6267, 62672Y-62672Y-10 (2006).

[13] Bonaglia, M., Pinna, E., Puglisi, A., Esposito, S., Guerra, J. C., Myers, R.., Dipper, N., "First cophasing of a segmented mirror with a Tunable Filter and the Pyramid wavefront sensor," 77392Y-77392Y-10 (2010).

[14] Parks, R. E., Evans, C.., Shao, L., “Calibration of Interferometer Transmission Spheres,” Opt. Fabr. Test. Work. OSA Tech. Dig. Ser., 80-83 (1998).

[15] Zhou, P.., Burge, J. H., "Limits for interferometer calibration using the random ball test," Proc. SPIE 7426, $74260 \mathrm{U}$ (2009). 